\title{
Tunneling voltage dependent heights of faulted and unfaulted Ir islands on $\operatorname{Ir}(111)$
}

\author{
C. Busse, ${ }^{1, *}$ S. Baud ${ }^{2}$ G. Bihlmayer ${ }^{3}$ C. Polop,${ }^{1}$ T. Michely,${ }^{1}$ and S. Blügel ${ }^{3, \dagger}$ \\ ${ }^{1}$ I. Physikalisches Institut der RWTH Aachen, 52056 Aachen, Germany \\ ${ }^{2}$ Laboratoire de Physique Moléculaire, UMR CNRS 6624, Université de Franche-Comté, 25030 Besançon Cedex, France \\ ${ }^{3}$ Institut für Festkörperforschung, Forschungszentrum Jülich, 52425 Jülich, Germany
}

(Received 10 June 2003; published 11 November 2003)

\begin{abstract}
Analysis of homoepitaxial growth on $\operatorname{Ir}(111)$ by scanning tunneling microscopy (STM) reveals that two different phases nucleate. We find islands in the regular face-centered cubic (fcc) stacking as well as in the hexagonal close-packed (hcp) stacking. Performing STM measurements on fcc and hcp areas shows an apparent, voltage dependent height difference of up to $6 \%$ of the regular layer distance. By applying first-principles calculations, the voltage dependent height difference can be attributed to the difference in the electronic structures of the two phases. The atoms in hcp stacking appear lower for a wide range of tunneling voltages, opposite to the actual relaxation.
\end{abstract}

DOI: 10.1103/PhysRevB.68.201401

PACS number(s): 68.55.-a, 68.35.Fx, 73.20.-r

On fcc(111), adatoms can adsorb on two nonequivalent threefold hollow adsorption sites, the fcc, and the hcp site. By occupying the fcc site a stacking sequence ABc (lower case denotes the additional layer) is induced, whereas adsorption on an hcp site leads to an $\mathrm{ABa}$ sequence and introduces a stacking fault into the system. Growth in the presence of stacking faults leads to twin crystallite formation, which get embedded in the regular matrix and incoherent twin boundaries evolve. ${ }^{1}$ Therefore the density of stacking faults is decisive for the quality of thin films. On the other hand, a stacking fault is not necessarily a "fault"; there are several systems where the energetically disfavored stacking shows desirable properties. An example is the magnetic multilayer system $\mathrm{Co} / \mathrm{Cu}(111)$, where fcc $\mathrm{Co}$ has superior magnetic properties. Several studies address the problem of growing (metastable) fcc Co layers on $\mathrm{Cu}(111)$ (e.g., Refs. 2-4).

Some phenomenological studies of stacking-fault formation have been performed, ${ }^{5-10}$ but only recently understanding of the underlying atomic processes was achieved in the model system $\operatorname{Ir} / \operatorname{Ir}(111) .{ }^{11}$ The equilibrium distribution of small clusters between hep and fcc is frozen in by the attachment of immobilizing adatoms during growth.

A dependence of the electronic structure on the stacking geometry was first observed in the system $\mathrm{Ag} / \mathrm{Ag}(111)$ by low-energy electron microscopy. ${ }^{6}$ In the heteroepitaxial system $\mathrm{Co} / \mathrm{Cu}(111)$ this effect was analyzed in detail and explained by differences in the local density of states. ${ }^{12}$ Differences in electronic structure have also been observed between the hcp and fcc phases of the $\mathrm{Au}(111)$ herringbone reconstruction, ${ }^{13}$ but in this system they arise from the change in the band structure due to an electronic superlattice.

In the present study we observed an apparent, tunneling voltage dependent height difference between homoepitaxial Ir-adatom islands in fcc and in hcp stacking. This effect can be explained on the basis of the different electronic structure of the two phases as calculated by ab initio calculations.

The experiments were performed in a UHV chamber with a base pressure $P<3 \times 10^{-11}$ mbar. The sample was cleaned by repeated cycles of sputtering and annealing, resulting in a clean surface and a terrace width of several $1000 \AA$. Prior to deposition the sample was flashed to a temperature ensuring desorption of all species that might have adsorbed from the background gas. Ir was evaporated from a resistance-heated wire. Special care was exercised to ensure clean deposition conditions $\left(P<1 \times 10^{-10} \mathrm{mbar}\right)$. Scanning was performed in constant current mode $(I=1 \mathrm{nA})$. The bias voltage $U$ is given with respect to the sample, i.e., for $U<0 \mathrm{~V}$ the sample is more negative than the tip.

During homoepitaxial growth on $\operatorname{Ir}(111)$ stacking-fault islands nucleate under a wide range of deposition temperatures $T$ and deposition rates $F$. At the temperature under concern here $(T=350 \mathrm{~K})$, the ratio of the probability $P_{\text {hcp }}$ to find an island in the hcp stacking to the probability $P_{\text {fcc }}$ to find it in the fcc stacking is $\left(P_{\mathrm{hcp}} / P_{\mathrm{fcc}}\right)=0.12 .{ }^{11}$ Upon further growth the sparsely distributed hcp islands become immersed in the surrounding regular fcc matrix. A representative morphology is shown in Fig. 1(a). In the percolated layer an area in hcp stacking is surrounded by fcc phase; the phase boundary between the two phases can be identified by the roughly triangular gap. Occasionally this gap becomes decorated by a row of single atoms as indicated by the box in Fig. 1(a). Details on growth in the presence of stacking-faults and especially on the formation mechanism of the decoration rows will be given in a forthcoming publication.

The different stacking geometries become clearly visible in Fig. 1(b). Here the area marked by a box in Fig. 1(a) is
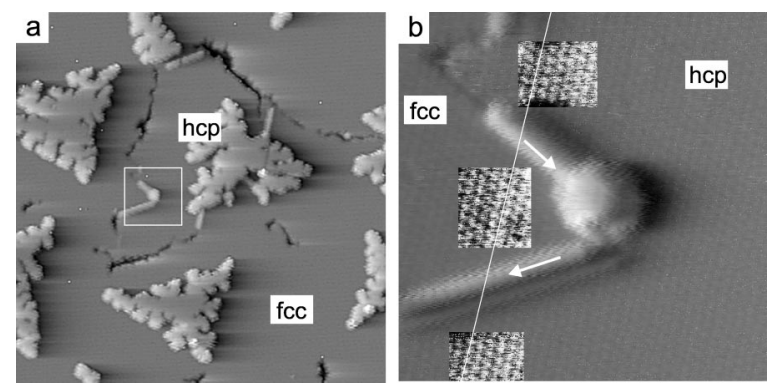

FIG. 1. STM topograph of hcp and fcc phases created by deposition of $2.2 \mathrm{ML}$ at $T=350 \mathrm{~K}$ with $F=1.5 \times 10^{-2} \mathrm{ML} / \mathrm{s}$. (a) Picture size $550 \AA \times 550 \AA$. (b) Picture size $80 \AA \times 80 \AA$. The contrast was enhanced locally to show the atomic resolution in the hcp and in the fcc phase. Arrows indicate the orientation of the decoration rows. 

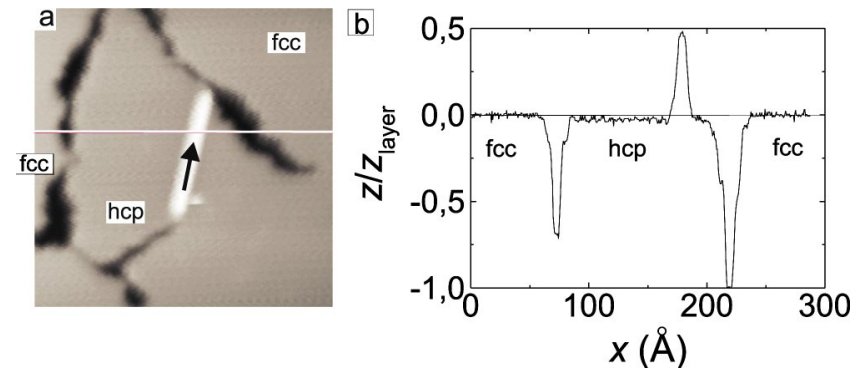

FIG. 2. (a) STM topograph showing hep and fcc areas after deposition of $0.91 \mathrm{ML}$ at $T=350 \mathrm{~K}$ with $F=1.6 \times 10^{-2} \mathrm{ML} / \mathrm{s}$, greyscale image, picture size $240 \AA \times 240 \AA$. The arrow indicates the orientation of the decoration row. (b) Linescan along the white line in (a), tunneling voltage $U=1.2 \mathrm{~V}$, tunneling current $I$ $=1.0 \mathrm{nA}$, constant current mode. The height difference between fcc and hcp is $\Delta z=0.03 z_{\text {layer }}$, with $z_{\text {layer }}$ the distance between two consecutive (111) layers in the Ir crystal, $z_{\text {layer }} \approx 2.22 \AA$.

imaged with atomic resolution. By doing this for the whole hcp phase an atomic grid was superimposed on the image, where the atoms in the hcp phase sit at the crossing points of the grid lines. The fcc phase is almost completely surrounded by the hcp phase, so it is possible to extrapolate this grid also to the fcc area. The grid fits with the atomic positions in the hcp phase, but does not fit in the fcc phase. Here, the atoms are not situated on the crossing points of the mesh, but rather in the up triangles, as it is expected for a stacking-fault area. This is illustrated by one line of the grid shown in Fig. 1(b). This specific line is not singularly positioned to fit the atoms, but rather picked out of the optimized grid. This line is running on top of the atoms in the hcp phase, but between the atomic rows in the fcc phase.

The decoration rows can be used to determine the respective stacking in situations where rather complicated morphologies are studied. We attribute a direction to the rows by choosing as a convention that this direction is given with fcc to the right and hcp to the left-hand side [see arrows in Fig. 1(b)]. Only rows in [1 $\overline{1} 0],[\overline{1} 01]$, and [01 $\overline{1}]$ are observed, i.e., only in three of the six dense-packed directions on fcc(111). The crystallographic orientation of the sample is known. This allows us to unambiguously identify the respective stacking in the presence of decoration rows.

In Fig. 2(a) one can see a grayscale picture of an area containing both phases, which are identified by the presence of the bright decoration rows as introduced above. The linescan across this morphology along the white line is shown in Fig. 2(b). Close inspection of the scan reveals that the hcp phase is imaged lower than the fcc phase. For the tunneling conditions applied in this scan (tunneling voltage $U$ $=1.2 \mathrm{~V}$, tunneling current $I=1.0 \mathrm{nA}$, constant current mode) this height difference is $0.03 z_{\text {layer }}$, with $z_{\text {layer }}$ the distance between two consecutive (111) layers in the Ir crystal, $z_{\text {layer }}=\sqrt{1 / 3} a \approx 2.22 \AA$, with $a=3.84 \AA$ the bulk lattice constant for Ir. Although the noise on the scanline is clearly visible the large number of points taken on the fcc as well as the hcp phase lead to a small error in the overall height difference.

The electronic nature of this effect becomes apparent

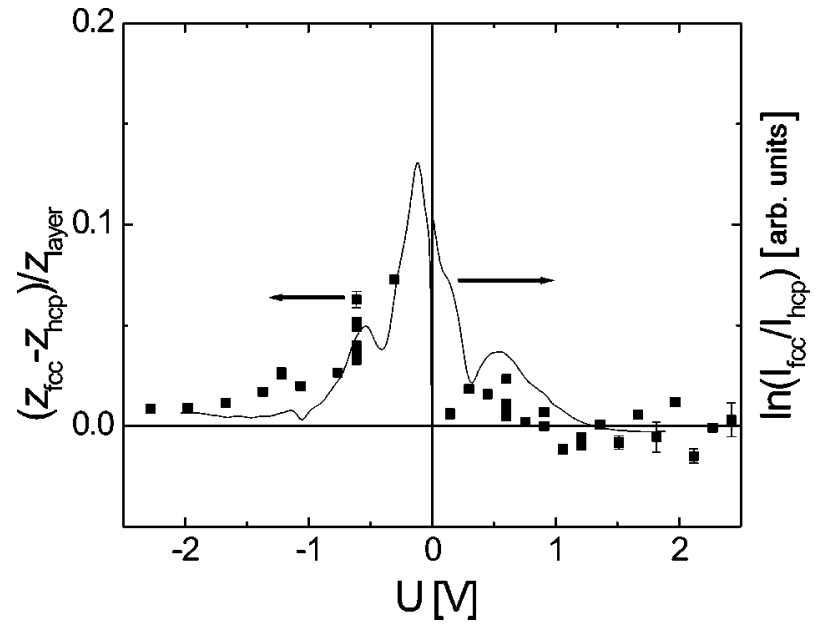

FIG. 3. Dependence of the relative height difference $\left(z_{\mathrm{fcc}}\right.$ $\left.-z_{\text {hcp }}\right) / z_{\text {layer }}$ on tunneling voltage $U$. ( $\left.\mathbf{\square}\right)$ : Experimental results obtained by voltage dependent STM, $I=1 \mathrm{nA}$. Full line: result obtained by $a b$ initio calculations.

upon analyzing linescans obtained with different bias voltages (Fig. 3). In all the experiments evaluated here the coverage was around 1 monolayer (ML). Here the scatter is mainly due to changing tip conditions. Nevertheless, a clear structure is visible: Starting with the fcc phase imaged higher for high negative bias voltages, the height difference goes through a maximum for small negative bias voltages $U$. A second smaller maximum is visible for small positive voltages, until with increasing $U$ we observe a change in sign for $\left(z_{\mathrm{fcc}}-z_{\mathrm{hcp}}\right)$, i.e., now the hcp phase is imaged higher.

The apparent height difference between fcc and hcp must be caused by differences in the electronic structures of the regular fcc crystal and an fcc crystal with one hcp layer on top of it. In order to relate the voltage dependent behavior of the scanning tunneling microscopy (STM) images to the structural identity of Ir islands, we performed electronic structure calculations based on the density-functional theory (DFT) in the local-density approximation of von Barth and Hedin. ${ }^{14}$ The results were obtained with the full-potential linearized augmented plane-wave method in bulk and film geometry, ${ }^{15,16}$ as implemented in the computer code FLEUR. ${ }^{17}$ On the scale relevant to the electronic structure the hcp and fcc islands have a rather large lateral extension of $\approx 100 \AA$ (Ref. 18) and are therefore modeled by perfect $\operatorname{Ir}(111)$ surfaces with and without stacking fault of the final surface layer. Both surfaces are described by nine layer films with inversion symmetry embedded in infinite vacuum at both sides of the film. All total energy results presented are calculated using about 100 basis functions per atom. The forces exerted on the atoms were minimized to optimize the structure down to a maximum force of $0.5 \mathrm{meV} / \mathrm{a}$.u. per atom. For the integration over the Brillouin-zone (BZ) we used a special $\mathbf{k}_{\|}$-point set of 57 points within the irreducible wedge (1/12) of the two-dimensional BZ. The calculations of bulk Ir were performed with $216 \mathbf{k}$ points in the irreducible wedge of the three-dimensional BZ.

We determined the theoretical Ir bulk lattice constant to $3.81 \AA$ which is in good agreement with the experimental value of $3.84 \AA$. Using the theoretical bulk lattice constant, 
both Ir surfaces were structurally optimized by including the interlayer relaxations of the first three layers on both sides of each film for both systems. The interlayer relaxations are small, but slightly different for the surface with and without stacking fault. Relative to the ideal bulk truncation, we found inward relaxation for both surfaces (fcc terminated and hcp terminated). The fcc surface layer is relaxed inwards by $0.025 \AA\left(0.0115 z_{\text {layer }}\right)$ and the hcp surface layer is relaxed inwards by $0.01 \AA\left(0.0048 z_{\text {layer }}\right)$. Thus the atoms in the hcp terminated surface relax inwards less by $0.015 \AA$. The energy of the surface with the stacking fault is $90.4 \mathrm{meV}$ higher than the ideal surface, a value close to the results of Hamilton et al. ${ }^{19}$ who found a difference of $81 \mathrm{meV}$. The higher binding energy of the regular layer is something expected since the equilibrium structure of Ir is fcc. Furthermore, this explains by a simple bond strength-bond length argument why the more weakly bound hcp layer relaxes less inward than the stronger bound fcc layer. However, this is not what is observed in STM topographs like the one depicted in Fig. 2, as the hcp phase appears lower for most bias voltages.

According to the model of Tersoff and Hamann ${ }^{20}$ to describe the tunneling current measured in STM, the variation of the tunneling current $I$ with the bias voltage $U$ is proportional to the local density of states (LDOS) of the sample in the vacuum $n$ at the tip position $\boldsymbol{r}=\left(\boldsymbol{r}_{\|}, z\right)$, i.e., $d I / d U$ $\propto n\left(\boldsymbol{r} \mid E_{F}+e U\right)$, where $E_{F}$ is the Fermi energy, and $\left(\boldsymbol{r}_{\|}, z\right)$ are the lateral $\left(\boldsymbol{r}_{\|}\right)$and vertical $(z)$ tip coordinates (the tipsample distance is measured from the position of the nucleus of the surface atom). Thus the energy integrated LDOS (ILDOS) of occupied $(U<0)$ or unoccupied $(U>0)$ states in the energy range $\left(E_{F}, E_{F}+e U\right)$ contribute to the tunneling current, $I(\boldsymbol{r}) \propto \int_{E_{F}}^{E_{F}+e U} n(\boldsymbol{r} \mid E) d E$.

The experiments evaluated to obtain Fig. 3 were carried out without atomic resolution. Thus the relevant quantity is the laterally averaged local density of states $\bar{n}(z \mid E)$ defined as $\bar{n}(z \mid E)=1 / A_{\|} \int_{A_{\|}} n\left(\boldsymbol{r}_{\|}, z \mid E\right) d \boldsymbol{r}_{\|}$, where $A_{\|}$is the area of the surface unit cell. The average tunneling current $\bar{I}(z, U)$ can then be computed from $\bar{n}(z \mid E)$ as

$$
\bar{I}(z, U) \propto \int_{E_{F}}^{E_{F}+e U} \bar{n}(z \mid E) d E .
$$

This quantity can be directly compared with the current measured in the constant-current STM images. ${ }^{23}$ From our experiences with comparing theoretically determined STM images with the experimental ones, we think that a good comparison can be achieved using the model of Tersoff and Hamann for a bias-voltage range of $\pm 2 \mathrm{eV} .{ }^{21}$

Figure 4 shows the LDOS in the vacuum $\bar{n}$ for the hcp and fcc terminated $\operatorname{Ir}(111)$ surfaces at a tip-sample distance of $z_{\text {ref }}=9.41 \AA$. The position of the sample atoms was taken to be the average of the positions of the surface atoms of the fcc and hcp surfaces. The energies are given with respect to the Fermi level. The work functions of the fcc and hcp surfaces are $W_{\text {fcc }}=6.306 \mathrm{eV}$ and $W_{\mathrm{hcp}}=6.374 \mathrm{eV}$, respectively. Therefore the Fermi levels are shifted by $70 \mathrm{meV}$ with respect to the vacuum zero. The higher LDOS for fcc than for

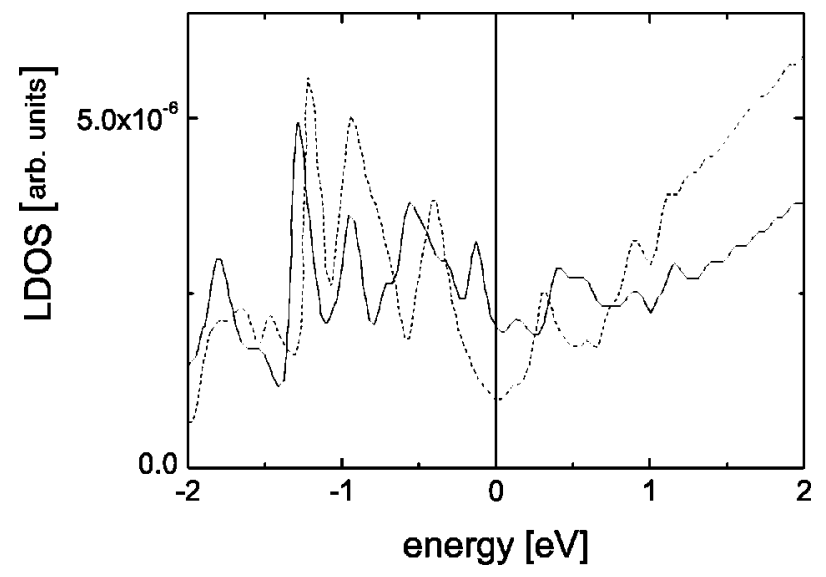

FIG. 4. Local density of states (LDOS) in the vacuum $\bar{n}$ for the fcc (full line) and hcp (dashed line) terminated $\operatorname{Ir}(111)$ surfaces at a tip-sample distance of $z=9.41 \AA$.

hcp close to the Fermi level offers a straightforward interpretation of the observation that the fcc phase is imaged higher for voltages around $U=0 \mathrm{~V}$ (see Fig. 3). To reach the same tunneling current on the hcp phase as on the fcc phase, the tip has to be closer to the sample in order to compensate for the lower LDOS.

This argument can be turned in to a quantitative estimation of the height difference between the fcc and hcp phases using the LDOS, or according to Eq. (1) the tunneling current $\bar{I}\left(z_{\text {ref }}\right)$ at a reference height $z_{\text {ref }}$. We assume an exponential dependence of the tunneling current with the tipsample distance, $\bar{I}(z, U)=\bar{I}\left(z_{\text {ref }}, U\right) e^{-\kappa\left(z-z_{\text {ref }}\right)}$ at arbitrary $z$. Actually, this behavior can be explicitly found in the calculated ILDOS's for different $z_{\text {ref }}$. Transforming the above equation into $z-z_{\text {ref }}=(1 / \kappa) \ln \left[\bar{I}\left(z_{\text {ref }}, U\right) / \bar{I}(z, U)\right]$ and taking into account that different currents are measured above the fcc and the hcp phases we can write for the height difference

$$
z_{\mathrm{fcc}}(U, \bar{I})-z_{\mathrm{hcp}}(U, \bar{I})=\frac{1}{\bar{\kappa}} \ln \left(\frac{\bar{I}_{\mathrm{fcc}}\left(z_{\mathrm{ref}}, U\right)}{\bar{I}_{\mathrm{hcp}}\left(z_{\mathrm{ref}}, U\right)}\right)-\frac{\Delta \kappa}{\bar{\kappa}} \Delta z_{\mathrm{ref}} .
$$

We imposed $\bar{I}_{\mathrm{fcc}}\left(z_{\mathrm{fcc}}, U\right)=\bar{I}_{\mathrm{hcp}}\left(z_{\mathrm{hcp}}, U\right)$ due to the constant current mode employed. $\Delta z_{\text {ref }}$ denotes the difference between the average $\left(z_{\mathrm{fcc}}+z_{\mathrm{hcp}}\right) / 2$ and $z_{\text {ref }} . \Delta \kappa(\bar{\kappa})$ is the difference between (average of) $\kappa_{\mathrm{fcc}}$ and $\kappa_{\mathrm{hcp}}$. Ignoring the correction term $\propto \Delta z_{\text {ref }}$ for a moment, the height difference is proportional to the logarithm of the tunneling currents at an arbitrary reference height. These reference currents are known via Eq. (1). Since the approximation of the exponential decay of the tunneling current with distance is not perfect, it is best to choose a $z_{\text {ref }}$ close to the real height of the tip in the STM measurements. The height difference can thus be calculated from the values obtained for $\bar{n}\left(z_{\text {ref }}\right)$ in the DFT calculations. Of course, the exact $z_{\text {ref }}$ is not known from the experiment and we fixed $z_{\text {ref }}=9.41 \mathrm{AA}$. The deviation of best $z_{\text {ref }}$, chosen as the unknown voltage dependent average $\left(z_{\mathrm{fcc}}+z_{\mathrm{hcp}}\right) / 2$, from the fixed value causes an uncertainty in $\Delta z=-(\Delta \kappa / \bar{\kappa}) \Delta z_{\text {ref }} \simeq\left(W_{\text {fcc }}-W_{\text {hcp }}\right) /\left(W_{\text {fcc }}+W_{\text {hcp }}\right) \Delta z_{\text {ref }}$. 
Since the difference between the work functions $W$ of the fcc and hcp stacked system is small, even an error in $z_{\text {ref }}$ of $4 \AA$ causes only an error in $\Delta z$ of $0.01 z_{\text {layer }}$. In Fig. 3, the calculated and measured quantities are compared. The calculated curve is in good qualitative agreement with the measurements, reproducing the shape of the curve including the maxima and the change in sign for high voltages. One remarkable result is that even though the hcp surface shows less inward relaxation, the LDOS indicates an apparent outward relaxation of the fcc surface in the STM images. It is rather surprising that such large differences between the two phases exist since hcp and fcc stackings differ only in the second nearest-neighbor interaction and, in opposite to the $\mathrm{Cu}(111)$ surface, no gap in the surface band structure gives rise to distinct surface states.

The difference in the electronic structures between the two phases can be used to distinguish them also in situations where the stacking cannot be inferred from the presence of decoration rows or from the island shape (compare Refs. 11 12). One can speculate that similar effects may be found on other surfaces as well. For example, in the recent experiments on $\mathrm{Cu} / \mathrm{Cu}(111)$ (Ref. 10) such a criterion could offer a more straightforward way to determine the respective stacking than the effect of the just slightly different equilibrium shapes of the islands.

Summarizing, even though the hcp surface shows a smaller inward relaxation, the difference in the LDOS leads to the effect that in the STM image the hcp surface appears higher than the fcc surface for a wide range of tunneling parameters. Comparing the STM images with ab initio calculations, we find that the theoretically predicted and experimentally observed dependence of the apparent height difference on tunneling voltage agree quite well. The contrast change with bias voltage as well as the apparent height difference are of electronic origin. Voltage dependent STM combined with $a b$ initio calculations provides a strategy to identify structurally different homoepitaxial islands in the system $\operatorname{Ir}(111)$ and may be applicable to other systems as well.

We acknowledge experimental help of L. Athanatos. This work was supported by the Deutsche Forschungsgemeinschaft via the project "Atomare Prozesse beim homoepitaktischen Schichtwachstum unter extremen Nichtgleichgewichtsbedingungen."
*Corresponding author. Electronic address: busse@physik.rwthaachen.de

'On Leave from the Department of Physics, University of Osnabrück, 49046 Osnabrück, Germany.

${ }^{1}$ M.J. Stowell, Epitaxial Growth (Academic Press, New York, 1975), Vol. B.

${ }^{2}$ M. Zheng, J. Shen, C.V. Mohan, P. Ohresser, J. Barthel, and J. Kirschner, Appl. Phys. Lett. 74, 425 (1999).

${ }^{3}$ P. Le Fevre, H. Magnan, O. Heckmann, V. Briois, and D. Chandesris, Phys. Rev. B 52, 11462 (1995).

${ }^{4}$ J. Camarero, L. Spendeler, G. Schmidt, K. Heinz, J.J. de Miguel, and R. Miranda, Phys. Rev. Lett. 73, 2448 (1994).

${ }^{5}$ K. Meinel, M. Klaua, and H. Bethge, Phys. Status Solidi A 110, 189 (1988).

${ }^{6}$ J. Tersoff, A.W. Denier van der Gon, and R.M. Tromp, Phys. Rev. Lett. 72, 266 (1994).

${ }^{7}$ S.A. de Vries, W.J. Huisman, P. Goedtkindt, M.J. Zwanenburg, S.L. Bennet, and E. Vlieg, Phys. Rev. Lett. 81, 381 (1998).

${ }^{8}$ J. Camarero, J. de la Figuera, J.J. de Miguel, R. Miranda, J. Alvarez, and S. Ferrer, Surf. Sci. 459, 191 (2000).

${ }^{9}$ H.A. van der Vegt, J. Álvarez, X. Torrelles, S. Ferrer, and E. Vlieg, Phys. Rev. B 52, 17443 (1995).
${ }^{10}$ M. Giesen and H. Ibach, Surf. Sci. 529, 135 (2003).

${ }^{11}$ C. Busse, C. Polop, M. Müller, K. Albe, U. Linke, and T. Michely, Phys. Rev. Lett. 91, 056103 (2003).

${ }^{12}$ A.L. Vázquez de Parga, F.J. García-Vidal, and R. Miranda, Phys. Rev. Lett. 85, 4365 (2000).

${ }^{13}$ W. Chen, V. Madhavan, T. Jamneala, and M.F. Crommie, Phys. Rev. Lett. 80, 1469 (1998).

${ }^{14}$ U.V. Barth and L. Hedin, J. Phys. C 5, 1629 (1972).

${ }^{15}$ E. Wimmer, H. Krakauer, M. Weinert, and A.J. Freeman, Phys. Rev. B 24, 864 (1981).

${ }^{16}$ M. Weinert, E. Wimmer, and A.J. Freeman, Phys. Rev. B 26, 4571 (1982).

${ }^{17}$ See http://www.flapw.de

${ }^{18}$ C. Busse, W. Langenkamp, C. Polop, H. Hansen, U. Linke, P.J. Feibelman, and T. Michely, Surf. Sci. Lett. 539, L560 (2003).

${ }^{19}$ J.C. Hamilton, M.R. Sorensen, and A.F. Voter, Phys. Rev. B 61, R5125 (2000).

${ }^{20}$ J. Tersoff and D.R. Hamann, Phys. Rev. B 31, 805 (1985).

${ }^{21}$ S. Heinze, R. Abt, S. Blügel, G. Gilarowski, and H. Niehus, Phys. Rev. Lett. 83, 4808 (1999).

${ }^{22}$ S. Heinze, S. Blügel, R. Pascal, M. Bode, and R. Wiesendanger, Phys. Rev. B 58, 16432 (1998).

${ }^{23}$ The quantity $\bar{n}$ is the first star $n^{(1)}$ in Ref. 22 . 